\title{
Quark stars admitting a one-parameter group of conformal motions
}

\author{
M. K. Mak* and T. Harkd \\ Department of Physics, The University of Hong Kong, Pokfulam Road, Hong Kong
}

(Dated: January 13, 2004)

\begin{abstract}
An exact analytical solution describing the interior of a charged strange quark star is found under the assumption of spherical symmetry and the existence of a one-parameter group of conformal motions. The solution describes a unique static charged configuration of quark matter with radius $R=9.46 \mathrm{~km}$ and total mass $M=2.86 M_{\odot}$.
\end{abstract}

\section{INTRODUCTION}

It is largely believed today that the strange quark matter, consisting of the $\mathrm{u}, \mathrm{d}$ and $\mathrm{s}$ quarks is the most energetically favorable state of baryon matter. Witten [1] specified the two ways of formation of the strange matter: the quarkhadron phase transition in the early universe and conversion of neutron stars into strange ones at ultrahigh densities. Quark bag models in the theories of strong interactions suppose that the breaking of physical vacuum takes place inside hadrons. As a result the vacuum energy densities inside and outside a hadron become essentially different and the vacuum pressure $B$ on a bag wall equilibrates the pressure of quarks thus stabilizing the system.

The structure of a realistic strange star is quite complicated and can be described as follow 2]. Beta-equilibrated strange quark - star matter consists of an approximately equal mixture of up, down and strange quarks, with a slight deficit of the latter. The Fermi gas of $3 A$ quarks constitutes a single color-singlet baryon with baryon number A. This structure of the quarks leads to a net positive charge inside the star. Since stars in their lowest energy state are supposed to be charge neutral, electrons must balance the net positive quark charge in strange matter stars. Being bounded by the Coulomb force, rather than the strong one, as is the case for quarks, the electrons extend several hundred fermis beyond the surface of the strange star. Associated with this electron displacement is a very strong electric dipole layer that can support, out of contact with the surface of a strange star, a crust of nuclear material, which it polarizes. The neutron drip density determines the maximal possible density at the base of the crust (the inner crust density) 2]. Being electrically charge neutral the neutrons do not feel the Coulomb force and hence would gravitate toward the quark core where they become converted into strange quark matter.

The theory of the equation of state of strange stars is directly based on the fundamental QCD Lagrangian [3]

$$
L_{Q C D}=\frac{1}{4} \sum_{a} F_{\mu \nu}^{a} F^{a \mu \nu}+\sum_{f=1}^{N_{f}} \bar{\psi}\left(i \gamma^{\mu} \partial_{\mu}-g \gamma^{\mu} A_{\mu}^{a} \frac{\lambda^{a}}{2}-m_{f}\right) \psi
$$

where the subscript $f$ denotes the various quark flavors $u, d, s, c$ etc. and the nonlinear gluon field strength is given by

$$
F_{\mu \nu}^{a}=\partial_{\mu} A_{\nu}^{a}-\partial_{\nu} A_{\mu}^{a}+g f_{a b c} A_{\mu}^{b} A_{\nu}^{c} .
$$

QCD predicts a weakening of the quark-quark interaction at short distances

Assuming that interactions of quarks and gluons are sufficiently small the energy density $\varepsilon$ and pressure $p$ of a quark -gluon plasma at temperature $T$ and chemical potential $\mu_{f}$ can be calculated by thermal theory. Neglecting quark masses in first order perturbation theory, the equation of state is given by [1], [2]:

$$
\varepsilon=\sum_{i=u, d, s, c ; e^{-}, \mu^{-}} \varepsilon_{i}+B, p+B=\sum_{i=u, d, s, c ; e^{-}, \mu^{-}} p_{i},
$$

where $B$ is the difference between the energy density of the perturbative and non-perturbative QCD vacuum (the bag constant). Hence the pressure - energy density relation for quark matter is given by the MIT bag model equation of state [1], 2]

$$
p=\frac{1}{3}(\rho-4 B) .
$$

\footnotetext{
*Electronic address: mkmak@vtc.edu.hk

${ }^{\dagger}$ Electronic address: harko@hkucc.hku.hk
} 
Equation (4) is essentially the equation of state of a gas of massless particles with corrections due to the QCD trace anomaly and perturbative interactions. These are always negative, reducing the energy density at given temperature by about a factor two when $\alpha_{s}=0.5[3]$.

Most of the investigations of the stellar quark-gluon plasma have been done under the assumption of the electric charge neutrality of the quark-gluon plasma that reads $\sum_{i=u, d, s, c ; e^{-}, \mu^{-}} q_{i} n_{i}=0$. In the case of a star formed from massless $\mathrm{u}, \mathrm{d}$ and $\mathrm{s}$ quarks the charge neutrality condition can be explicitly formulated as $2 n_{u} / 3=\left(n_{d}+n_{s}\right) / 3[2]$.

There are several proposed mechanisms for the formation of quark stars. Quark stars are expected to form during the collapse of the core of a massive star after the supernova explosion as a result of a first or second order phase transition, resulting in deconfined quark matter [4]. The proto-neutron star core or the neutron star core is a favorable environment for the conversion of ordinary matter to strange quark matter 4]. Another possibility is that some neutron stars in low-mass X-ray binaries can accrete sufficient mass to undergo a phase transition to become strange stars [5]. This mechanism has also been proposed as source of radiation emission for cosmological $\gamma$-ray bursts $[6]$.

It is the purpose of the present Letter to consider the behavior of strange quark stars described by the MIT bag model with respect to one class of admissible transformations: conformal motions. We shall also explore the physical consequences on the structure and properties of the strange stars of the existence of this transformation.

As a group of admissible transformations for a strange star we shall consider spherically symmetric and static distributions of quark matter that admits a one-parameter group of conformal motions, i.e.

$$
L_{\xi} g_{i k}=\phi(r) g_{i k}
$$

where the left-hand side is the Lie derivative of the metric tensor describing the interior gravitational field of a strange star with respect to the vector field $\xi^{i}$ and $\phi(r)$ is an arbitrary function of the radial coordinate r. This type of symmetry has been intensively used to describe the interior of neutral or charged general relativistic stellar-type objects [7] [ 8], 9]. With the assumption (5) the gravitational field equations describing the interior of a strange star can be integrated in Schwarzschild coordinates and an exact simple physical solution, corresponding to a charged strange star, can be obtained.

The present paper is organized as follows. In Section II we obtain the general solution for the Einstein-Maxwell equations for a static charged configuration with the matter content obeying the bag model equation of state under the assumption that the space-time admits a one-parameter group of conformal motions. In Section III we discuss and conclude our results.

\section{STRANGE QUARK STARS ADMITTING CONFORMAL SYMMETRY}

In the following we shall adopt geometrized units such that $8 \pi G=c=1$. The sign conventions used are those of the Landau-Lifshitz timelike convention. Let us consider a spherically symmetric static distribution of strange quark matter. In Schwarzschild coordinates the line element takes the following form:

$$
d s^{2}=e^{\nu(r)} d t^{2}-e^{\lambda(r)} d r^{2}-r^{2}\left(d \theta^{2}+\sin ^{2} \theta d \chi^{2}\right)
$$

The total energy-momentum tensor $T_{i}^{k}$ inside the strange star is assumed to be the sum of two parts $M_{i}^{k}$ for the quark matter and $E_{i}^{k}$ for an electromagnetic contribution, respectively: $T_{i}^{k}=M_{i}^{k}+E_{i}^{k}$.

The energy-momentum tensor for the quark matter has the usual expression

$$
M_{i}^{k}=(\rho+p) u_{i} u^{k}-p \delta_{i}^{k}
$$

with $p$ and $\rho$ related by the bag model equation of state (4). In equation (7) $u^{i}=\delta_{0}^{i} e^{-\nu / 2}$ is the four-velocity satisfying $u_{i} u^{i}=1$.

The electromagnetic contribution is given by

$$
E_{i}^{k}=-\frac{1}{4 \pi}\left(F_{i l} F^{k l}-\frac{1}{4} \delta_{i}^{k} F_{l m} F^{l m}\right),
$$

where $F_{i k}$ is the electromagnetic field tensor defined in terms of the four-potential $A_{i}$ as

$$
F_{i k}=A_{i, k}-A_{k, i}
$$

For the electromagnetic field we shall adopt the gauge $A_{i}=(\varphi(r), 0,0,0)$.

The Einstein-Maxwell equations describing the interior of a charged strange star can be expressed as

$$
R_{i}^{k}-\frac{1}{2} \delta_{i}^{k} R=T_{i}^{k}, F_{i k, l}+F_{l i, k}+F_{k l, i}=0, F_{; k}^{i k}=-j^{i} / 2,
$$


where $j^{i}=\bar{\rho}_{e} u^{i}$ is the four-current density and $\bar{\rho}_{e}$ is the proper charge density.

Using the line element (6) the field equations (10) take the form (we denote the derivative with respect to $r$ by a prime):

$$
\begin{gathered}
\rho+E^{2}=-e^{-\lambda}\left(\frac{1}{r^{2}}-\frac{\lambda^{\prime}}{r}\right)+\frac{1}{r^{2}}, \\
-p+E^{2}=-e^{-\lambda}\left(\frac{\nu^{\prime}}{r}+\frac{1}{r^{2}}\right)+\frac{1}{r^{2}}, \\
p+E^{2}=\frac{1}{2} e^{-\lambda}\left(\nu^{\prime \prime}+\frac{\left(\nu^{\prime}\right)^{2}}{2}+\frac{\nu^{\prime}-\lambda^{\prime}}{r}-\frac{\nu^{\prime} \lambda^{\prime}}{2}\right), \\
\frac{d}{d r}\left(r^{2} E\right)=\frac{1}{2} \rho_{e} r^{2} .
\end{gathered}
$$

In equations (11)-(14) $E$ is the usual electric field intensity defined as $E^{2}=-F_{01} F^{01}$ and $E(r)=e^{-(\nu+\lambda) / 2} \varphi^{\prime}(r)$ with $\varphi^{\prime}(r)=F_{10}$. The charge density $\rho_{e}$ in equation (14) is related to the proper charge density $\bar{\rho}_{e}$ by $\rho_{e}=\bar{\rho}_{e} e^{\lambda / 2}$. We first integrate equation (14) to obtain $E(r)=Q(r) / r^{2}$, where $Q(r)=\frac{1}{2} \int_{0}^{r} \rho_{e} r^{2} d r=\frac{1}{2} \int_{0}^{r} \bar{\rho}_{e} e^{\lambda / 2} r^{2} d r$ is the charge within radius $r$.

Now we shall assume that the space-time describing the interior of the strange quark star admits a one-parameter group of conformal motions (5), i.e.

$$
L_{\xi} g_{i k}=\xi_{i ; k}+\xi_{k ; i}=\phi(r) g_{i k}
$$

with $\phi(r)$ an arbitrary function of $r$.

Using the line element (6) equation (15) explicitly reads

$$
\xi^{i} \nu^{\prime}=\phi, \xi^{0}=\bar{C}=\text { const. }, \xi^{1}=\phi r / 2, \lambda^{\prime} \xi^{1}+2 \frac{d \xi^{1}}{d r}=\phi .
$$

Equations (16) have the general solution given by []

$$
e^{\nu}=A^{2} r^{2}, \phi=C e^{-\lambda / 2}, \xi^{i}=\bar{C} \delta_{0}^{i}+\phi r \delta_{1}^{i} / 2,
$$

with $A$ and $C$ arbitrary constant of integration.

Hence the requirement of the existence of conformal motions imposes strong constraints on the form of the metric tensor coefficients for a strange star. Substituting (17) into the field equations (11)-(14) we obtain

$$
\rho+E^{2}=\frac{1}{r^{2}}\left(1-\frac{\phi^{2}}{C^{2}}\right)-\frac{2}{C^{2}} \frac{\phi \phi^{\prime}}{r},-p+E^{2}=\frac{1}{r^{2}}\left(1-3 \frac{\phi^{2}}{C^{2}}\right), p+E^{2}=\frac{1}{C^{2}} \frac{\phi^{2}}{r^{2}}+\frac{2}{C^{2}} \frac{\phi \phi^{\prime}}{r} .
$$

We can formally solve the field equations (18) and express $p, \rho$ and $E^{2}$ as

$$
\rho=-\frac{3 Y^{\prime}}{2 r}+\frac{1}{2 r^{2}}, p=\frac{Y^{\prime}}{2 r}+\frac{4 Y-1}{2 r^{2}}, E^{2}=\frac{Y^{\prime}}{2 r}+\frac{1-2 Y}{2 r^{2}},
$$

where we have introduced a new variable $Y=\frac{\phi^{2}}{C^{2}}$.

From equations (19) we obtain, by using the bag equation of state of the strange matter (4), the following differential equation for $Y$ :

$$
\frac{d Y}{d r}=-\frac{2 Y}{r}+\frac{2}{3 r}-\frac{4 B}{3} r
$$


Eq. (20) has the general solution given by

$$
Y(r)=\frac{1}{r^{2}}\left[C_{1}+\frac{r^{2}}{3}\left(1-B r^{2}\right)\right]
$$

with $C_{1}$ an arbitrary integration constant.

In order to obtain finite and well-defined values of the energy density and mass for all $r \leq R$ (we denote by $R$ the radius of the strange star) it is necessary that the arbitrary integration constant $C_{1}$ be zero. Therefore we obtain the following explicit exact solution describing the interior of a charged strange star:

$$
e^{\nu}=A^{2} r^{2}, e^{\lambda}=\frac{3}{1-B r^{2}}, \rho=\frac{1}{2 r^{2}}+B, p=\frac{1}{6 r^{2}}-B, E^{2}=\frac{1}{6 r^{2}} .
$$

The radius $R$ of the static strange quark matter configuration can be obtained from the condition of the vanishing pressure at the surface of the star, $p(R)=0$ and is given by $R=1 / \sqrt{6 B}$. Using the results $E=Q(r) / r^{2}$, $E^{2}=1 /\left(6 r^{2}\right)$ and $R=1 / \sqrt{6 B}$, we obtain the charge distribution in the interior of the quark star in the form $Q=r / \sqrt{6}=\sqrt{B} R r$. The charge is linearly increasing with the radius $r$.

The solution of the Einstein-Maxwell equations for $r>R$ is given by the Reissner-Nordstrom metric as

$$
d s^{2}=\left(1-2 M / r+Q^{2} / r^{2}\right) d t^{2}-\left(1-2 M / r+Q^{2} / r^{2}\right)^{-1} d r^{2}-r^{2}\left(d \theta^{2}+\sin ^{2} \theta d \chi^{2}\right),
$$

where $M$ and $Q$ are the total mass and charge of the strange star respectively. To match the line element (6) with the Reissner-Nordstrom metric across the boundary at $r=R$ we require the continuity of the gravitational potentials and of the radial electric field at $r=R$. The continuity of $e^{\nu}$ leads to the determination of the constant $A$ as $A^{2}=\left(1-2 M / R+Q^{2} / R^{2}\right) / R^{2}$, while the continuity of the electric field leads to a total charge-radius relation of the form $Q(R)=R / \sqrt{6}=\sqrt{B} R^{2}$. The intensity of the electric field on the surface of the strange star is given by $E(R)=Q(R) / R^{2}=\sqrt{B}$. The continuity of on the boundary $r=R$ leads to a mass charge relation of the form $M=2 Q^{2}(R) /(3 R)+R / 3$. On the other hand the total mass of the quark matter component of the star is given by

$$
M_{q}=\frac{1}{2} \int_{0}^{R} \rho r^{2} d r=B R^{3} / 6+R / 4 .
$$

The distribution of the proper charges density inside the quark star follows from equations (11) and (14) and is given by $\bar{\rho}_{e}=\sqrt{2\left(1-B r^{2}\right)} /\left(3 r^{2}\right)$. Immediately, by taking $r=R=1 / \sqrt{6 B}$, the proper surface charge density is given by $\bar{\rho}_{e}(R)=\sqrt{\frac{20}{3}} B$.

Hence we have obtained the complete solution of the gravitational field equations for a charged strange quark star described by the MIT bag model.

\section{DISCUSSIONS AND FINAL REMARKS}

A physically acceptable interior solution of the gravitational field equations must comply with the following conditions: a) the matter density $\varepsilon$ and the fluid pressure p should be non-negative throughout the distribution b) the gradients $d \rho / d r$ and $d p / d r$ should be negative c) the speed of sound should not exceed the speed of light as implication of causality fulfillment d) the interior metric should match continuously with an exterior solution.

The solution to the gravitational field given by equations (22) satisfies all these four criteria, since we have, $\rho \geq 0$, $p \geq 0, d \rho / d r=-1 / r^{3}<0$ and $d p / d r=-1 /\left(3 r^{3}\right)<0$ for all $0 \leq r \leq R$. The speed of sound is given by $v_{s}=c / \sqrt{3}$. The singularity in the charge density and mass density is physically acceptable since the total charge and mass are finite.

The strange star model with conformal motions describes a single stable quark matter configuration with radius given by $R=c / \sqrt{48 \pi G B}=9.46 \mathrm{~km}$ and with the mass of the quark matter $M_{q}=4 \pi B R^{2} / 3+4 \pi R c^{2} /(16 \pi G)=$ $40 \pi B R^{3} / 3 \approx 3.545 \times 10^{33} \mathrm{~g} \approx 1.772 M_{\odot}$, where $B=10^{14} \mathrm{~g} / \mathrm{cm}^{3}$ and $M_{\odot}=2 \times 10^{33} \mathrm{~g}$ have been used. The total mass of the star (including the electromagnetic contribution) is $M=2.86 M_{\odot}$.

A complete description of static strange stars has been obtained based on numerical integration of mass continuity and TOV (hydrostatic equilibrium) equations for different values of the bag constant. Using numerical methods the maximum gravitational mass $M_{\max }$, the maximum baryon mass $M_{B, \max } \equiv 1.66 \times 10^{-27} \mathrm{~kg} \times N_{B}\left(N_{B}\right.$-the total baryon 
number of the stellar configuration) and the maximum radius $R_{\max }$ of the strange star, have been obtained, as a function of the bag constant, in the form [1], 10], 11], 12], 13]:

$$
M_{\max }=\frac{1.9638 M_{\odot}}{\sqrt{B_{60}}}, M_{B, \max }=\frac{2.6252 M_{\odot}}{\sqrt{B_{60}}}, R_{\max }=\frac{10.172 k m}{\sqrt{B_{60}}},
$$

where $B_{60} \equiv B /\left(60 \mathrm{MeV} \mathrm{fm}^{-3}\right)$.

Hence the presence of charge leads to a considerable increase in the mass of a stellar object obeying the MIT bag model equation of state. On the other hand the radius of the charged configuration is smaller than the maximum radius of the uncharged strange star.

The requirement that the strange quark star obeying the MIT equation of state admits the group of conformal motions is the simplest way that leads to an exact quark star model. But this requirement also necessities, in order to close the gravitational field equations, the introduction of an extra term in the energy-momentum tensor. In order to obtain a complete physical solution, especially one satisfying condition d) of physical acceptability in the present Letter we have supposed that the extra-term in the energy-momentum tensor is due to the presence of a static charge distribution inside the strange quark star. Other choices are also possible by adding to the energy-momentum tensor of the perfect quark fluid extra terms that corresponds to a magnetic field, possible anisotropic stresses related to some corrections to the MIT bag model state equation, super-fluidity etc. The arbitrariness in adding the extra term to the energy -momentum tensor also leads to the possibility of minimizing the contribution of the non-perfect fluid term. On the other hand a linearly increasing charge density resulting in a highly charged strange star surface is not excluded by realistic quark star models [2].

If $B=0$ the solution (22) is identical with the solution to the gravitational field equations obtained by Misner and Zapolsky, describing neutral matter obeying a $\gamma$-law equation of state 14].

Whether the strange star model presented in this paper actually describes a well-determined stellar structure can only be decided once reliable knowledge about the mechanisms that characterize strange and neutron star formation becomes available from the underlying theory of stellar evolution and from observational data.

[1] Witten E., Phys. Rev D 30, 272, 1984.

[2] Cheng K. S., Dai Z. G. and Lu T., Int. J. Mod. Phys. D 7, 139, 1998.

[3] Weinberg S., The Quantum Theory of Fields, (Cambridge University Press, Cambridge), volume II, 1996.

[4] Dai Z. G., Peng Q. H. and Lu T., Astrophys. J. 440, 815, 1995.

[5] Cheng K. S. and Dai Z. G., Phys. Rev. Lett. 77, 1210, 1996.

[6] Cheng K. S. and Dai Z. G., Phys. Rev. Lett. 80, 18, 1998.

[7] Herrera L. and Ponce de Leon J., J. Math. Phys. 26, 2303, 1985.

[8] Herrera L. and Ponce de Leon J., J. Math. Phys. 26, 2018, 1985.

[9] Herrera L. and Ponce de Leon J., J. Math. Phys. 26, 778, 1985.

[10] Alcock C., Farhi E., Olinto A., Astrophys. J. 310, 261, 1986

[11] Haensel P., Zdunik J. L., Schaeffer R., Astron. Astrophys. 160, 121, 1986.

[12] Haensel P., Zdunik J. L., Nature 340, 617, 1989.

[13] Gourgoulhon E., Haensel P., Livine R., Paluch E., Bonazzola S., Marck J.-A., Astron. Astrophys. 349, $851,1999$.

[14] Misner C. W. and Zapolsky H. S., Phys. Rev. Lett. 12 , 635, 1964. 